\title{
Microbiota and Bone Health: The Gut-Musculoskeletal Axis
}

\author{
René Rizzoli ${ }^{1}$
}

Received: 7 January 2018 / Accepted: 11 January 2018 / Published online: 9 February 2018

(c) Springer Science+Business Media, LLC, part of Springer Nature 2018

The largest number of cells $\left(10^{\mathrm{E}} 14\right)$ in the human body are located within the intestinal tract lumen. This number is much higher than the number of cells in any human body parenchyma. The estimated number of different species varies between 1000 and 5000 comprising gram-positive and gram-negative bacteria, Archaea, Eukarya, as well as viruses. These organisms are collectively called the gut microbiota (GM). They mostly refer to the large intestine content, but all parts of the gastrointestinal tract are colonized with an increasing density from the duodenum to the distal colon. GM is now considered as an organ modulating the expression of genes involved in mucosal barrier function, immune system, food digestion, and energy metabolism [1]. The microbiome refers to the aggregate collection of genomes and genes present in gut microbiota. Dysbiosis is a state of microbial imbalance in the gut. It refers to a change in microbiota structure and/or function [2]. Gnotobiotic animals are animals raised under germ-free conditions or under germ-free conditions and then colonized with well-defined and controlled microbes.

Gut microbiota can vary according to age [3], sex, diet, living conditions [4], diseases requiring antibiotics treatment [5], and drugs [6]. Over the last 12 years, GM has gathered increasing interest because of associations or even causal relationships as suggested or demonstrated for a large variety of disorders including bowel inflammatory diseases, irritable bowel syndrome, colo-rectal cancer, obesity, metabolic syndrome, type 2 diabetes, atherosclerosis, non-alcoholic fatty liver, malnutrition, asthma, autoimmune diseases, psoriasis, multiple sclerosis, infections, anxiety, depression, and autism. A link with GM has also been recognized for periodontitis, rheumatoid arthritis, and spondyloarthropathies. Bone homeostasis has also been shown to be influenced by GM composition or production [7, 8]. In this special issue

René Rizzoli

rene.rizzoli@unige.ch

1 Service of Bone Diseases, Geneva University Hospitals and Faculty of Medicine, 1211 Geneva 14, Switzerland of the Journal, the associations between bone health and GM are reviewed and discussed.

In a well-detailed paper, Schwarzer and Strigini et al. are reviewing the role of GM in growth, through experimental models including Drosophila, worms, crustacea, Zebrafish, chicken, and mice [9]. One factor implicated in bone growth is insulin-like growth factor I (IGF-I). GM is able to modulate and stimulate IGF-I synthesis by the host in a dynamic interaction, as summarized by Yan and Charles [10]. A GM-host interaction contributes to control the host immune system development. A series of GM-produced factors influence immune cells, suggesting a link between dysbiosis and autoimmune, metabolic, and degenerative disorders as reviewed by d'Amelio and Sassi [11]. By modulating the immune system, GM is becoming a new player in the regulators of bone turnover and function. Considering the interplay between bone physiology, gastrointestinal tract content, microbiology, and immunology, Ohlsson and Sjögren are proposing the term "Osteomicrobiology" to cover this field where GM changes are capable of preventing ovariectomy-induced bone loss [12]. Such a cross-talk appears to exist between GM and skeletal muscle too. Grosicki et al. are summarizing the evidence for a role of GM in the age-related changes in skeletal muscle mass, structure, and function [13]. GM composition, metabolism, and production can be modified by either prebiotics, which are non-digestible fibers metabolized in the distal part of the gastrointestinal tract, or probiotics, which are micro-organisms conferring a health benefit on the host when administered in adequate amounts. These topics are extensively discussed in two specific reviews $[14,15]$. Finally, a possible source of both prebiotics and probiotics is fermented dairy products, which in addition provide calcium, protein, phosphorus, and various micronutrients. Their role in bone growth and in age-related bone loss is summarized in the last article of this special issue [16].

Gut microbiota appears to be a major player in the various determinants of musculoskeletal health. However, it remains to be demonstrated in well-conducted long-term randomized controlled trials, whether interventions changing 
GM composition or function are capable of preventing bone loss and/or reducing fracture risk. Genetic background, sex, dietary intakes, and living conditions are likely important confounding factors necessary to consider in evaluating the effects of GM changes on musculoskeletal health.

\section{References}

1. Human Microbiome Project Consortium (2012) Structure, function and diversity of the healthy human microbiome. Nature 486:207-214. https://doi.org/10.1038/nature11234

2. Gordon JI (2012) Honor thy gut symbionts redux. Science 336:1251-1253. https://doi.org/10.1126/science.1224686

3. Biagi E, Nylund L, Candela M et al (2010) Through ageing, and beyond: gut microbiota and inflammatory status in seniors and centenarians. PLoS ONE 5:e10667. https://doi.org/10.1371/journ al.pone. 0010667

4. Claesson MJ, Cusack S, O'Sullivan O et al (2011) Composition, variability, and temporal stability of the intestinal microbiota of the elderly. Proc Natl Acad Sci USA 108(Suppl 1):4586-4591. https://doi.org/10.1073/pnas.1000097107

5. Cho I, Blaser MJ (2012) The human microbiome: at the interface of health and disease. Nat Rev Genet 13:260-270. https://doi. org/10.1038/nrg3182

6. Forslund K, Hildebrand F, Nielsen T et al (2015) Disentangling type 2 diabetes and metformin treatment signatures in the human gut microbiota. Nature 528:262-266. https://doi.org/10.1038/natur e15766
7. Weaver CM (2015) Diet, gut microbiome, and bone health. Current Osteoporos Rep 13:125-130. https://doi.org/10.1007/s1191 4-015-0257-0

8. Hernandez CJ, Guss JD, Luna M, Goldring SR (2016) Links between the microbiome and bone. J Bone Miner Res 31:16381646. https://doi.org/10.1002/jbmr.2887

9. Schwarzer M, Strigini M, Leulier F (2018) Gut microbiota and host juvenile growth. Calcif Tissue Int. https://doi.org/10.1007/ s00223-017-0368-y

10. Yan J, Charles JF (2018) Gut microbiota and IGF-1. Calcif Tissue Int. https://doi.org/10.1007/s00223-018-0395-3

11. D’Amelio P, Sassi F (2018) Gut microbiota, immune system, and bone. Calcif Tissue Int. https://doi.org/10.1007/s0022 3-017-0331-y

12. Ohlsson C, Sjogren K (2018) Osteomicrobiology: a new cross-disciplinary research field. Calcif Tissue Int. https://doi.org/10.1007/ s00223-017-0336-6

13. Grosicki GJ, Fielding RA, Lustgarten MS (2018) Gut microbiota contribute to age-related changes in skeletal muscle size, composition, and function: biological basis for a gut-muscle axis. Calcif Tissue Int. https://doi.org/10.1007/s00223-017-0345-5

14. Whisner CM, Castillo LF (2018) Prebiotics, bone and mineral metabolism. Calcif Tissue Int. https://doi.org/10.1007/s0022 3-017-0339-3

15. McCabe LR, Parameswaran N (2018) Advances in probiotic regulation of bone and mineral metabolism. Calcif Tissue Int. https:// doi.org/10.1007/s00223-018-0403-7

16. Rizzoli R, Biver E (2018) Effects of fermented milk products on bone. Calcif Tissue Int. https://doi.org/10.1007/s0022 3-017-0317-9 